\title{
Service Aggregation Using Relational Operations on Interface Parameters
}

\author{
George Feuerlicht \\ Faculty of Information Technology, \\ University of Technology, Sydney, \\ P.O. Box 123 Broadway, Sydney, NSW 2007, Australia \\ jiri@it.uts.edu.au
}

\begin{abstract}
Many practitioners recommend the use of coarse-grained services that minimize the number of messages and avoid the need to maintain state information between invocations. However, when considered from a software engineering perspective, coarse-grained services suffer from a number of significant drawbacks, including limited reuse and difficult composability. An important challenge for the developers of service-oriented applications is to determine appropriate level of service granularity to ensure that services are reusable and at the same time have good performance characteristics. Decisions about service granularity need to be taken in the context of a methodological framework rather than using ad hoc heuristics. In this paper we describe a method for service aggregation that uses relational operations over interface parameters to assemble services from low granularity atomic service operations. We illustrate the impact of service aggregation on cohesion and coupling using examples and discuss service granularity in the context of application requirements.
\end{abstract}

Keywords: service design, service aggregation, relational operations.

\section{Introduction}

Many researchers regard SOA (Service Oriented Architecture) as a message-based paradigm and view service-oriented applications as orchestrations of message exchanges that facilitate service interactions [1]. A frequently used argument in favor of message-orientation is that coarse-grained services (i.e. services with aggregated message payloads) avoid the need to maintain state information between service invocations, simplifying recovery in the event of failure. Furthermore, it is argued that coarse-grained services achieve performance advantages by reducing the number of network interactions required to implement a given business function. Asynchronous message-oriented patterns that characterize SOA alleviate many of the issues associated with synchronous, RPC-based protocols and provide an alternative approach for the implementation of failure-resilient and highly scalable distributed applications. However, when considered from a software engineering perspective, coarse-grained services suffer from a number of significant drawbacks, including limited reuse and poor composability [2]. 
A key determinant of service reuse is service granularity, i.e. the scope of functionality that individual services implement. There is an inverse relationship between service granularity and service reusability; as the scope of functionality implemented by a given service increases, the potential for reuse diminishes. Coarse-grained services are associated with complex message structures that often contain embedded business rules, context information, and instructions for processing of the message. This is evident in various industry standard message specifications, for example, the OTA specification (Open Travel Alliance, www.opentravel.org/) that forms the basis for the implementation of Web Services for the travel industry. Using this specification airline flight booking is implemented with the message pair OTA_AirBookRQ and OTA_AirBookRS. The flight booking request document OTA_AirBookRQ is a complex document that contains a large number of data elements (many optional) and includes flight booking, itinerary, and traveler and payment details. Decomposing the flight booking request into separate, lower-granularity operations such as flight enquiry, flight booking, and payment significantly increases potential for reuse (e.g. payment operation can be reused for car rental, or a hotel room booking). At the same time lower-granularity operations facilitate a more conversational interaction between the travel agent and the airline that more closely corresponds to the requirements of the travel booking business process. Also importantly, service granularity impacts on the ability to evolve service-oriented applications. Once externalized, the service interface must be maintained for the duration of the service lifetime to avoid invalidating existing applications that use the service. Coarse-grained services that externalize complex data structures exhibit high levels of data coupling and are difficult to evolve without producing undesirable side-effects.

The main challenge for the developers of service-oriented applications is to determine appropriate level of service aggregation to ensure that services are reusable, exhibit a high degree of mutual independence, and at the same time have good performance characteristics. Decisions about service granularity play a key role in the design of services, and need to be considered in the context of a methodological framework. In previous publications we have described a methodological framework for the design of services that uses top-down decomposition based on the data properties of interface parameters to maximize cohesion and minimize coupling of service operations [2], [3], [4]. In this paper we focus on the problem of service aggregation and extend the original design framework to include bottom-up service aggregation that uses relational operations to combine normalized service interfaces into composite service operations. We analyze the impact of service aggregation on cohesion and coupling and discuss service granularity in the context of application requirements.

In the following section (sections 2) we briefly summarize our method for service design and describe how complex business functions can be decomposed into elementary service operations with normalized interfaces. Section 3 illustrates the method using a Flight Enquiry example loosely based on the OTA specification. We then consider the problem of service granularity and describe a technique for service aggregation that uses relational operations over interface parameters to assemble higherlevel services from atomic service operations (section 4). In the concluding section 5 we briefly review related literature, and discuss the benefits of a data engineering approach to service design identifying potential for further work. 


\section{Service Design Method}

The design method consists of two main design stages. The first stage involves topdown decomposition with the objective of identifying elementary, reusable service operations. We provide a brief overview of our approach to service decomposition in this section; full description of the method is available in earlier publications [2], [3]. The second design stage is the focus of this paper and involves service aggregation with the aim of optimizing service granularity with respect to the requirements of a particular message interchange scenario, e.g. airline travel booking dialogue. The level of service aggregation can be also fine-tuned to reflect performance, state management and other related considerations; such considerations are outside the scope of this paper.

For the purpose of this analysis we adopt the Web Services model of services where service interfaces (i.e. the signature of services) consist of port types, operations, and message types. In general, service interface can consist of a number of operations that implement the service, but it is possible for a service interface to support only a single ProcessMessage operation [1]; this makes the design method applicable to both RPC and message-oriented approaches. We make no a priory assumptions about the implementation style (i.e. binding style, RPC or document) and interaction model (i.e. synchronous or asynchronous) as we regard such decisions as being orthogonal to the task of designing service interfaces. We do not consider nonfunctional service requirements and focus entirely on data properties of service interfaces, i.e. the properties of inbound and outbound messages that implement data flows between services and determine the level of service coupling.

To support the requirements for service reuse and evolution, services must be designed to maximize cohesion and minimize coupling of service operations [4]. Maximizing cohesion requires that a module (i.e. service operation) performs a single, atomic function. Importantly, a high level of service cohesion leads to orthogonality of services as functional overlap is minimized, or eliminated altogether. The requirement for minimization of coupling dictates that service interfaces consist of individual data parameters rather than more complex data structures such as classes or object references [5]. In order to avoid undesirable interdependencies, services cannot share higher level abstractions (e.g. classes) and use inheritance as the underlying reuse mechanism. Consequently, the reuse mechanism is limited to service aggregation, i.e. the assembly of composite services from elementary (atomic) services. Service assembly is a recursive process that continues until the appropriate level of service granularity that corresponds to a specific business requirement is reached. Furthermore, using individual data parameters rather than complex data structures as inbound and outbound messages in service interfaces enables the application of data engineering principles to further minimize coupling between services.

During the first design stage complex business functions are progressively decomposed into elementary functions and then mapped to corresponding candidate service operations. This approach is consistent with maximizing cohesion as elementary business functions typically accomplish a single conceptual task and exhibit high levels of cohesion. The decomposition of a high-level business function, e.g. airline flight booking business function, can be achieved by modeling the interaction between a travel agent and an airline using a Sequence Diagram [4]. Given the initial set of 
candidate service operations, further decomposition to maximize cohesion and minimize coupling can be achieved by applying data normalization to the interface data parameters [6], [7].

\section{Flight Enquiry Example}

We now illustrate our service design framework using an example of the Flight Enquiry business process based on the OTA airline availability request/response messages: OTA_Air_AvailRQ and OTA_Air_AvailRS. For the purpose of this example we make a number of simplifying assumptions and use a subset of the OTA message data elements to populate the service interfaces. Using the service decomposition approach described in section 2 and assuming functional dependencies FD1-FD5 between data parameters of the relevant service interfaces, the corresponding set of operations with normalized interfaces is shown in Table 1.

FD1: OriginLocation, DestinationLocation, DepartureDate $\rightarrow$ FlightNumber

FD2: FlightNumber $\rightarrow$ DepartureAirport, DepartureTime, ArrivalAirport, ArrivalTime

FD3: FlightNumber, DepartureDate $\rightarrow$ ArrivalDate

FD4: FlightNumber, DepartureDate, CabinType $\rightarrow$ Quantity

FD5: FlightNumber, DepartureDate, CabinType $\rightarrow$ BasicFare, BasicFareCode

Table 1. Normalized interfaces for the Flight Enquiry Service

\begin{tabular}{|l|l|l|l|}
\hline Business Function & Operation & Input Parameters & $\begin{array}{l}\text { Output Parame- } \\
\text { ters }\end{array}$ \\
\hline $\begin{array}{l}\text { Requests for avail- } \\
\text { able flights for a pair } \\
\text { of origin and destina- } \\
\text { tion cities on a given } \\
\text { departure date. }\end{array}$ & FlightEnquiry & $\begin{array}{l}\text { OriginLocation, Des- } \\
\text { tinationLocation, } \\
\text { DepartureDate }\end{array}$ & FlightNumber \\
\hline $\begin{array}{l}\text { Request for flight } \\
\text { schedule information } \\
\text { for a given flight } \\
\text { number. }\end{array}$ & ScheduleEnquiry & FlightNumber & $\begin{array}{l}\text { DepartureAirport } \\
\text { DepartureTime, } \\
\text { ArrivalAirport, } \\
\text { ArrivalTime }\end{array}$ \\
\hline $\begin{array}{l}\text { Request for arrival } \\
\text { information for a } \\
\text { given flight. }\end{array}$ & ArrivalEnquiry & $\begin{array}{l}\text { FlightNumber, } \\
\text { DepartureDate }\end{array}$ & ArrivalDate \\
\hline $\begin{array}{l}\text { Request for seat } \\
\text { availability informa- } \\
\text { tion for a given flight } \\
\text { and cabin type. }\end{array}$ & SeatEnquiry & $\begin{array}{l}\text { FlightNumber, } \\
\text { DepartureDate, } \\
\text { CabinType }\end{array}$ & Quantity \\
\hline $\begin{array}{l}\text { Request for pricing } \\
\text { information for a } \\
\text { given flight and } \\
\text { cabin type. }\end{array}$ & PriceEnquiry & $\begin{array}{l}\text { FlightNumber, } \\
\text { DepartureDate, } \\
\text { CabinType }\end{array}$ & $\begin{array}{l}\text { FareBasisCode, } \\
\text { BaseFare }\end{array}$ \\
\hline
\end{tabular}

We can now implement the Flight Enquiry business process as a dialogue between a travel agent and an airline as illustrated in Figure 1. It is not our intension to fully model the workflow of the Flight Enquiry process as our focus is on data properties of 
interface parameters and on aggregation of service operations based on data parameters. We simplify the dialogue by excluding alternative execution paths, for example the termination of the booking process due to unavailability of seats on a particular flight.

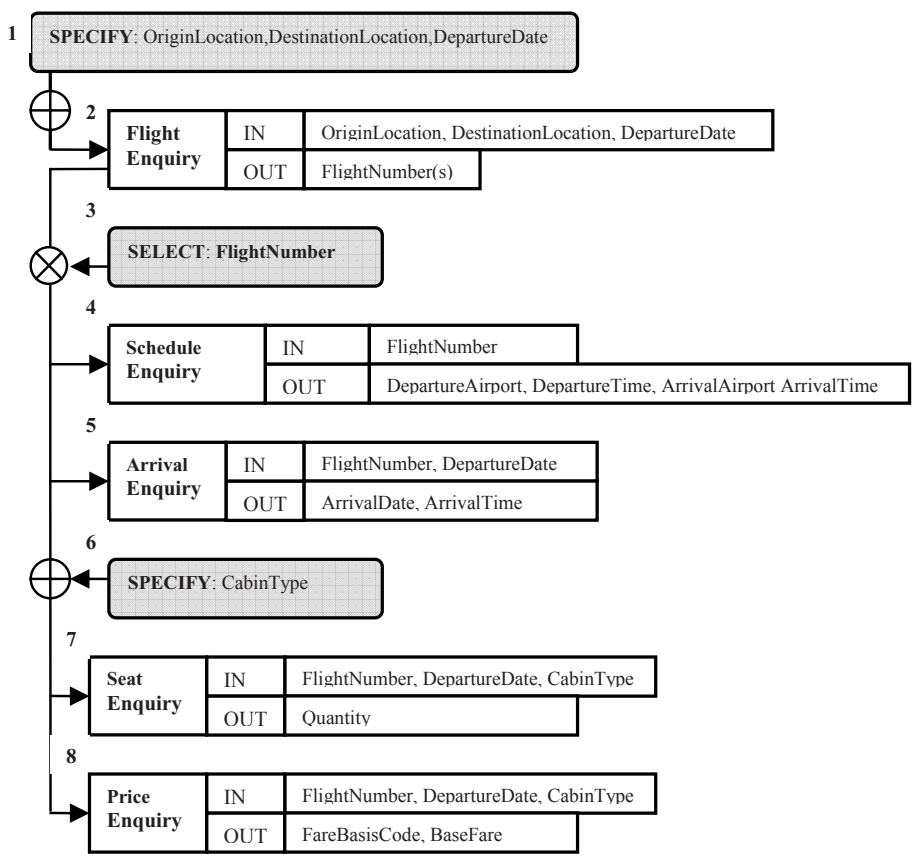

Fig. 1. Implementation of the Flight Enquiry process using elementary service operations

The dialogue proceeds as follows: The travel agent specifies input values for OriginLocation, DestinationLocation, and DepartureDate parameters (1). Following the execution of the FlightEnquiry operation (2), the travel agent selects a suitable flight (i.e. FlightNumber) (3). The travel agent then executes the operations ScheduleEnquiry (4) and ArrivalEnquiry (5) and supplies the value for CabinType, e.g. Economy (6). Finally, the travel agent executes the operations SeatEnquiry (7) and PriceEnquiry (8) to obtain the availability and price information, respectively.

\section{Service Aggregation Using Relational Operations}

As is evident from the above discussion, using fully normalized service interfaces results in fine-granularity operations with corresponding increase in the number of runtime calls and the complexity of the interaction dialogue. While the resulting interfaces produce a set of elementary and highly reusable services, they do not represent a practical solution. As an alternative the service designer may consider a coarse-granularity solution, supplying all user inputs (i.e. OriginLocation, 
DestinationLocation, DepartureDate, CabinType) at the beginning of the dialogue followed by the execution a single composite FlightEnquiry operation. As noted earlier (section 1) this leads to poor reuse and does not correspond to the inherently conversational dialogue that characterizes the flight enquiry business process. These fine-granularity and coarse-granularity alternatives represent two extreme design solutions. Finding a more optimal level of service granularity requires further examination, identifying operations that are suitable candidates for aggregations.

Let us now consider combining operations using interface parameters as the basis for service aggregation. It can be argued that the parameters of a normalized service interface constitute a relation with output parameters fully functionally dependent on the input parameter set, and can therefore be combined using relational operations (i.e. relational joins and union operations).

\subsection{Service Aggregation Using Join Operations}

We first consider aggregation of service operations based on common interface parameters using relational joins. Consider, for example combining operations SeatEnquiry and PriceEnquiry over common attributes (FlightNumber, DepartureDate, CabinType) producing a new operation SeatPriceEnquiry:

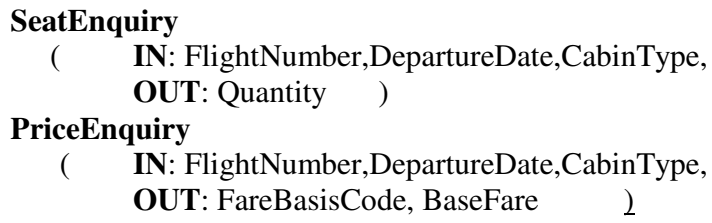

SeatPriceEnquiry $=$ SeatEnquiry NJN (FlightNumber, DepartureDate, CabinType) $_{\text {PriceEnquiry }}$

where $\mathbf{N J N}$ denotes a natural join, producing a new interface:

\section{SeatPriceEnquiry}

( IN: FlightNumber, DepartureDate, CabinType,

OUT: Quantity, FareBasisCode, BaseFare 2)

This solution leads to relative loss of cohesion as the resulting operation no longer implements a single atomic task, and in situations where it is used to perform a partial enquiry (e.g. seat availability enquiry only) the operation returns values that are not used by the application. The tradeoff can be justified in this instance on the basis that both operations are frequently performed together, and that the benefits of reduced number of operations and runtime procedure calls outweights the loss of cohesion. Similar considerations apply to combining operations ScheduleEnquiry and ArrivalEnquiry into a new operation TimeTable:

\section{ScheduleEnquiry}

IN: FlightNumber,

OUT: DepartureAirport, DepartureTime, ArrivalAirport, ArrivalTime ）

\section{ArrivalEnquiry}

\footnotetext{
( IN: FlightNumber, DepartureDate, OUT:ArrivalDate)
} 
The interface for the combined operation TimeTable can then be derived by joining the two interfaces based on the input parameter FlightNumber:

TimeTable $=$ ScheduleEnquiry $\mathbf{N J} \mathbf{N}_{\text {(FlightNumber) }}$ ArrivalEnquiry

\section{TimeTable}

IN: FlightNumber, DepartureDate,

OUT:DepartureAirport, DepartureTime, ArrivalAirport, ArrivalDate, ArrivalTime)

The aggregation of the ScheduleEnquiry and ArrivalEnquiry into a single operation TimeTable results in loss of cohesion, as the TimeTable operation is no longer strictly atomic; however, this can be justified on the basis that the TimeTable query closely corresponds to the requirements of the flight booking business process.

The resulting Flight Enquiry dialogue that uses the aggregated service operations is illustrated in Figure 2, and represent a solution that closely correspond to the conversational requirements of the flight booking business process. Further aggregation could now be considered, for example combining FlightEnquiry and TimeTable operations using the combination of FlightNumber, DepartureDate parameters, reducing the number of runtime calls with a corresponding loss of functional cohesion. Continuing the process of progressive aggregation to include SeatPriceEnquiry would result in a single, coarse granularity Flight Enquiry operation.

\subsection{Service Aggregation Using Union Operations}

Now consider a common requirement for combining flight availability information from multiple airlines, for example by executing the Flight Enquiry operation for two airlines: for KLM and BA:

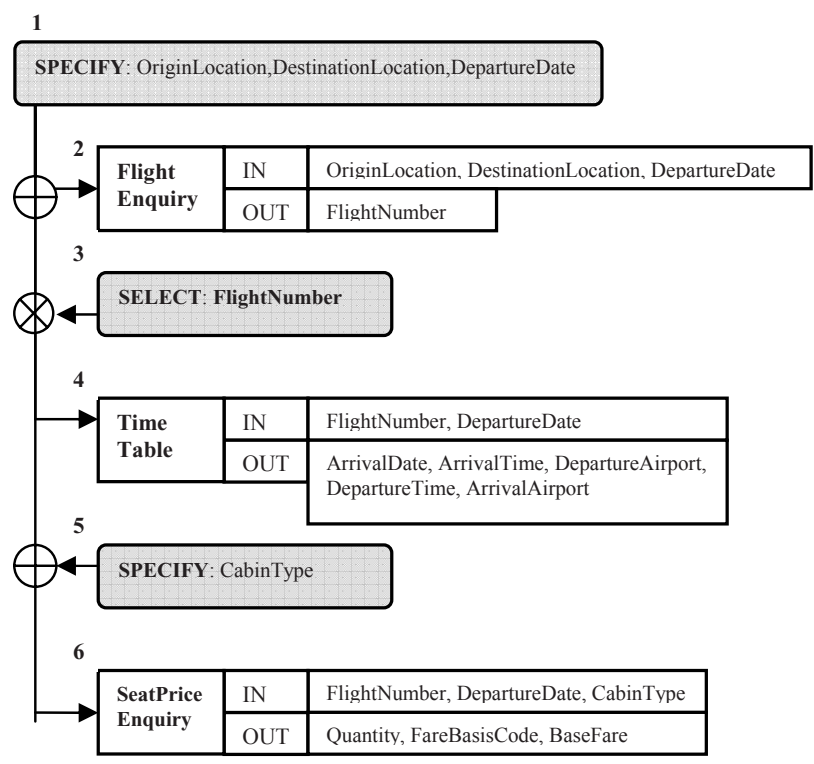

Fig. 2. Implementation of Flight Enquiry business process with aggregated service operations 


\section{FlightEnquiry}

( IN: OriginLocation, DestinationLocation, DepartureDate,

OUT: FlightNumber

This time the resulting interface is obtained using the union operation $\mathbf{U N}$ :

\section{FlightEnquiry = KLM_FlightEnquiry UN BA_FlightEnquiry}

The approach for aggregating service operations based on data properties of interface parameters described in this paper provides a framework for making decisions about the level of service granularity while considering the various design tradeoffs. The particular circumstances of a given application scenario would determine the level of service aggregation adopted by the designer. For example, an implementation in the context of fast and reliable local area network may use a fine-granularity solution with minimal aggregation of service operations.

\section{Related Work and Conclusions}

Most practitioners recommend the use of coarse-grained, message-oriented Web Services that minimize the number of messages and avoid the need to maintain state information between invocations. While it is evident that using fine-granularity service operations based on existing components leads to suboptimal design, excessive use of coarse-grained, document-centric services has its own limitations; in particular it results in poor reuse and undesirable interdependencies between services. It is therefore important that decisions about the level of service aggregation are made in the context of methodological framework, rather than based on ad hoc heuristics.

The study of service granularity is closely related to the work on services aggregation and composition. Service composition is an active research area with many diverse approaches being currently investigated. Industry based research views Web Services as abstract standardized interfaces to business processes and focuses on describing and implementing service composition using workflow specification languages such as BPEL. The Semantic Web research community takes a different approach and draws on AI planning research and run-time reasoning techniques based on ontological definitions of service semantics. A comprehensive review and comparison of the two approaches to service composition is provided in [8] and [9], with the conclusion that the Web Services composability problem remains essentially unsolved. In addition to BPEL, other standardization efforts include Web Services Choreography Interface (WSCI) and the Business Process Management Language (BPML) each taking a different approach to orchestration and choreography [10]. However, BPEL is today established as the industry standard language for the implementation of loosely coupled asynchronous business-to-business Web Services applications that share common XML data types and documents [11].

The approach described in this paper complements existing literature on the topic of design of services, proposing a methodological framework for making decisions about the level of service aggregation given specific application requirements and business process scenarios. We argue that both service aggregation and composition can be viewed as design-time activities that combine service operations based on data 
properties of interface parameters. Further research is needed to understand how service aggregation based on interface parameters can be used to achieve optimal service granularity given a set of application requirements. Another area of research interest concerns the application of this methodology in the more general context of services composition.

\section{References}

[1] Webber, J., Parastatidis, S.: Realising Service Oriented Architectures Using Web Services to be published in 2006 in Service Oriented Computing. MIT Press, Cambridge (2006)

[2] Feuerlicht, G.: Design of Service Interfaces for e-Business Applications using Data Normalization Techniques. In: Journal of Information Systems and e-Business Management, pp. 1-14. Springer, Heidelberg, ISS:1617-98 (2005)

[3] Feuerlicht, G.: Application of Data Engineering Techniques to Design of Messages Structures for Web Services. In: WDSOA'05. Proceedings of the First International Workshop on Design of Service-Oriented Applications, Amsterdam, The Netherlands, December 12, 2005, IBM Research Report RC23819 (W0512-29) (2005)

[4] Stevens, W.P., Myers, G.J., Constantine, L.L.: Structured Design. IBM Systems Journal 38(S2\&3) (1999)

[5] Myers, G.J.: Composite Structured Design, Van Nostrand Reinhold, 175 (1978) ISBN 0442-80584-5

[6] Codd, E.F.: Normalized Data Structure: A Brief Tutorial. In: Proceedings of 1971 ACMSIGFIDET Workshop on Data Description, Access and Control, San Diego, California, November 11-12, 1971, pp. 1-17. ACM Press, New York (1971)

[7] Date, C.J., Fagin, R.: Simple Conditions for Guaranteeing Higher Normal Forms in Relational Databases. ACM Transactions on Database Systems (TODS) 17(3), 465-476 (1992)

[8] Srivastava, B., Koehler, J.: Web Service Composition - Current Solutions and Open Problems. In: ICAPS (2003)

[9] Milanovic, N., Malek, M.: Current Solutions for Web Service Composition. IEEE Internet Computing 8(6), 51-59 (2004), http://dx.doi.org/10.1109/MIC.2004.58

[10] Peltz, C.: Web Services Orchestration and Choreography. Computer 36(10), 46-52 (2003)

[11] Pasley, J.: How BPEL and SOA Are Changing Web Services Development. IEEE Internet Computing 9(3), 60-67 (2005) 